Research Article

\title{
Propagation of Uncertain Events in Multilevel Handlings at Container Terminals from the Perspective of Hypernetwork
}

\author{
Bowei Xu $\mathbb{D}^{1},{ }^{1}$ Lingling Wang $\mathbb{D},{ }^{1}$ and Junjun $\mathrm{Li}\left(\mathbb{D}{ }^{2}\right.$ \\ ${ }^{1}$ Institute of Logistics Science \& Engineering, Shanghai Maritime University, Shanghai 201306, China \\ ${ }^{2}$ Merchant Marine College, Shanghai Maritime University, Shanghai 201306, China \\ Correspondence should be addressed to Bowei Xu; bwxu@shmtu.edu.cn
}

Received 31 December 2020; Revised 24 February 2021; Accepted 28 February 2021; Published 10 March 2021

Academic Editor: Jorge E. Macias-Diaz

Copyright (c) 2021 Bowei Xu et al. This is an open access article distributed under the Creative Commons Attribution License, which permits unrestricted use, distribution, and reproduction in any medium, provided the original work is properly cited.

Due to the complexity and dynamics of container terminal multilevel handlings, the occurrence of uncertain events often leads to the disruption of many operation plans and forms a chain reaction, resulting in economic losses and impacts. In this paper, the uncertain event propagation network model of container terminal multilevel handlings is constructed by using the hypernetwork theory, and the propagation influence of uncertain events between multilevel handlings at container terminals is considered. Through the topological characteristics of the uncertain event propagation network, the risk analysis is carried out for the relevance of nodes and the influence extent of uncertain events, and a simulation analysis is carried out to verify the validity of this model. The simulation results show that the model conforms to power law distribution, and the number of old nodes has an impact on the growth of hyperedge. The research provides a powerful tool for analysing the influence of uncertain events and their derivative events in the multilevel handlings at container terminals and has positive significance for reducing the losses caused by the changes of terminal plans and improving the operation efficiency.

\section{Introduction}

The multilevel handlings at automated container terminals are interactive each other (as shown in Figure 1). There is also "bullwhip effect" among all links in the container terminal handlings network. In fact, it is common for actual operation to deviate from the initial schedule due to various uncertain events.

The multilevel handlings at container terminals are dynamic, continuous, and diverse, but the actual handlings are complicated by various uncertain events. Uncertain events, such as ship arrival time delay, weather change, equipment failure, increase or decrease in handling capacity, and so on, will have an impact on the container terminals multilevel handlings and finally lead to plan changes. In addition, they also have chain effect. Congestion delay caused by uncertain events will be transferred among different nodes, causing other nodes to be delayed and suspended. The negative effects brought by uncertain events, such as increased time cost, idle equipment, and resources, increased container retention, delayed delivery, chaotic handling, and complex supply chain management, will also become more and more serious [1].

Focusing on the confusion of the container terminal operation plan caused by uncertain events, most scholars at home and abroad have conducted relevant research from the perspective of multilevel handlings scheduling. Tasoglu et al. [2] considered the dynamic arrival of ships and the integrated scheduling between berths and quay cranes (QCs) under random loading and unloading time and proposed a solution method based on simulation optimization. Xiang et al. [3] studied the influence of four uncertain conditions, including deviation of arrival time of ships, deviation of loading and unloading operation time of ships, unplanned arriving of ships, and failure of QCs, on the simultaneous allocation strategy of berths and QCs under discrete berths. Zhong et al. [4] considered the influence of integrated scheduling integrating QCs, automated guided vehicles (AGVs), and yard cranes (YCs). Ma et al. [5] studied the problems of unbalanced YCs works, shortage of YCs 


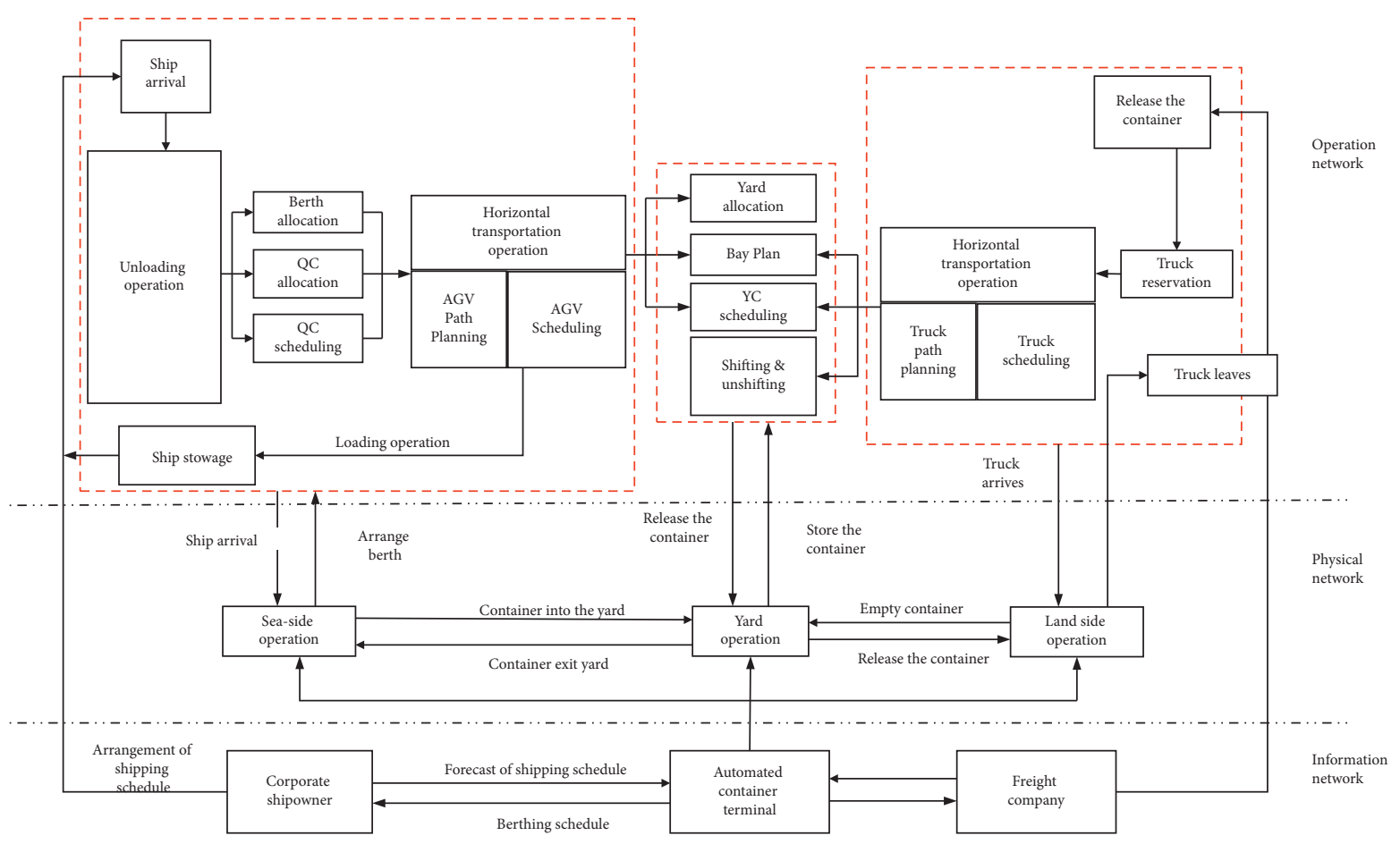

FIGURE 1: Schematic diagram of container terminal handlings.

resources during peak hours, and congestion of container terminals caused by random arrival time of trucks.

However, these studies did not consider the container terminal as a whole. Most studies focused on the scheduling of two or three handlings and did not consider the uncertain event propagation among the multilevel handlings at the container terminals [6-11]. Therefore, this paper takes container terminals as the study object and attempts to establish an uncertain event propagation network model based on the hypernetwork theory. The importance, relevance, and derivation of uncertain events and operation nodes are evaluated by means of the topological characteristics of hypernetwork. It helps port authorities effectively identify the risks of uncertain events, reduce the according losses, and improve the emergency response effect. The multilevel handlings at the container terminals are similar. They include the delivery/receipt of containers, the loading and unloading of yard cranes, the temporary storage of containers at the yard, the horizontal transportation of containers between berth and yard, the loading and unloading of gantry cranes, and the shipment of container vessel. Therefore, our model can be applied to different terminals. It provides a powerful tool for the correlation analysis among nodes in the propagation of uncertain events, as well as the derivation and risk assessment. The model is helpful to deeply analyse the propagation mechanism of uncertain events from the perspective of the interaction among the components of uncertain events. However, the current model only considers adding one new node per unit time in the process of hyperedge growth, and there may be multiple nodes in practice, so considering more nodes possibilities will be an improvement direction of this model.

\section{Analysis of Propagation Path of Uncertain Events}

2.1. Chain Propagation of Uncertain Events. The propagation effects of uncertain events and their derivative events in multilevel handlings at container terminals are mainly divided into the chain propagation and the network propagation. The chain propagation mainly describes the propagation among multilevel handlings; that is, the influence caused by an uncertain event will lead to the loss of other nodes in the multilevel handling through forward and reverse propagation. The forward propagation refers to the influence directly caused by this uncertain event, that is, the propagation direction from top to bottom along the flow sequence. As shown in Figure 2, the most direct impact caused by the bad weather is the ship delay and the suspension of handling. The ship delay will further lead to delivery delay, thus causing the supplier to bear economic losses. Reverse propagation mainly refers to the influence when an uncertain event produces a derivative event. In Figure 2, the delay of ship leads to the delay of loading and unloading operation, and the actual departure time of ship will also be delayed, and the delay will be brought to the next port. When the number of delayed ships in a container terminal reaches a certain number, it will lead to more serious port congestion, which will influence the operations of ships and container terminal. From Figure 2, the influence of uncertain events and their derivative events on multilevel handlings is mainly analysed from the longitudinal direction.

2.2. Network Propagation of Uncertain Events. The multilevel handlings at container terminals are closely connected. In addition, it often suffers diverse emergencies under complex 


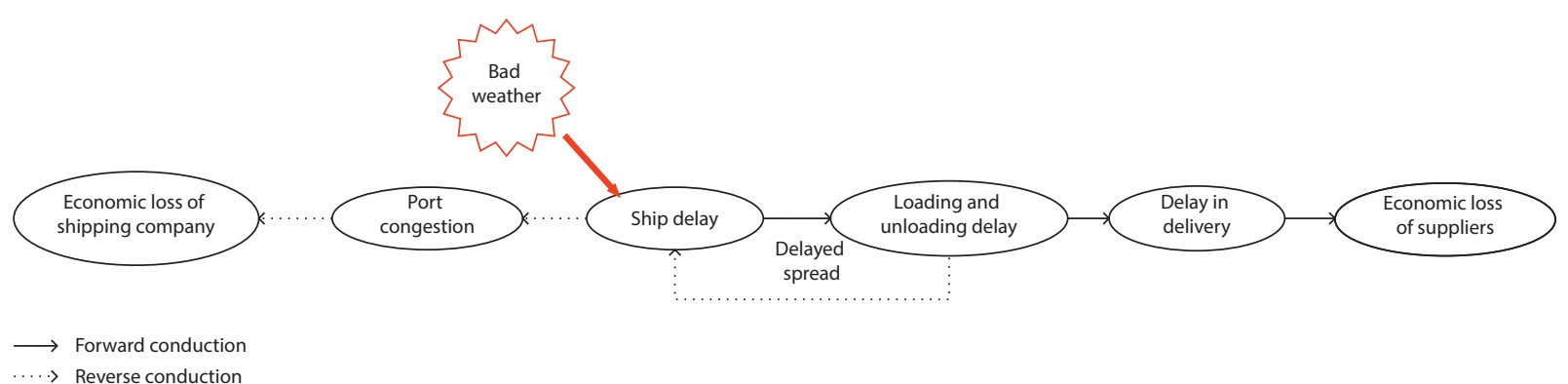

FIGURE 2: Example of chain propagation under sudden severe weather.

operation environment. As shown in Figure 3, when a normal node becomes a failed node after being attacked by an uncertain event, the impact of this propagation will become more and more serious over time and cause a wider spread. The collapse of a certain node will not only directly affect the superior and subordinate handlings but also affect other nodes that are not directly related but have correlations. The propagation speed and range of different uncertainties may be different. Multidimensional diffusion and superposition show strong network characteristics. These influences cross each other and overlap constantly, which makes the influence of propagation expand constantly.

\section{Uncertain Event Propagation Network Model Based on Hypernetwork}

3.1. Concept of Hypernetwork. The concept of hypernetwork was first put forward in 1985, and American scientist Nagurney Anna put forward that a network which is "higher but superior to the existing network" was called hypernetwork [12]. Hypernetwork is divided into three categories: hypergraph-based hypernetwork, multilayer optimized hypernetwork based on vibrational inequalities, and hypernetwork based on multisubnet integration. Hypernetwork based on hypergraph has a simple structure; it will be more suitable for describing the multistructure of the real world. Hypergraph is mainly used as a tool to connect multiple homogeneous or heterogeneous nodes through hyperedges, and thus the complex relationships among various nodes can be described concisely and accurately. This paper mainly studies hypernetwork based on hypergraphs.

The application and research of hypernetwork are mostly concentrated in the fields of knowledge learning, transportation network, balanced network, and so on [13-19]. The application of container terminals has not been tried yet. There are different levels of internal relations between different network propagation. Studying the propagation mechanism of uncertain events among container terminal handlings networks is of great positive significance to the arrangement, planning, and scheduling of container terminal multilevel handlings. It is an innovative choice to introduce hypernetwork theory into the uncertain event propagation at container terminals.

As shown in Figure 4, hypergraph-based hypernetwork can be expressed as graph $G=(V, E)$, where
$V=\left\{V_{1}, V_{2}, \ldots, V_{N}\right\}$ is node set, $V i$ represents a node of $G, N$ is the number of nodes in hypernetwork, and $E$ represents the set of hyperedges in hypernetwork $\left(V_{i}, V_{j} \in V, i \neq j\right)[20]$.

3.2. Topological Characteristics of Uncertain Event Propagation Network Based on Hypernetwork. In this paper, based on the topological characteristics of hypernetwork, the propagation influence of uncertain events at container terminals will be described. Taking the terminal facilities as nodes and the uncertain events and their derivative events as hyperedges, each node can be connected with the hyperedge. The main topological characteristics and meanings are analysed below [21].

3.2.1. Node Degree. The degree of a node in the hypernetwork is defined as the total number of nodes connected to other nodes through hyperedge. It represents the association times between a node and other nodes and reflects the association among the operations in each link. This relevance makes different handlings interact and influence each other and provides conditions for the diffusion and propagation of uncertain events. The more association between handlings, the wider the spread of events.

3.2.2. Node Hyperdegree. The hyperdegree of a node is defined as the number of hyperedges including the nodes. In an uncertain event propagation network, it indicates the number of uncertain events affecting this node. It reflects the superposition effect of uncertain events on nodes, and the node with higher hyperdegree means that this node is easily affected by multiple uncertain events, so port authorities should improve operation flexibility and pay more attention to this kind of node in daily operation planning.

3.2.3. Hyperedge Degree. The degree of a hyperedge refers to the number of hyperedges that share a common node with this hyperedge. In an uncertain event propagation network, some uncertain events will further lead to changes of other links' handling arrangement. When the affected extent of this node exceeds a certain threshold, a new uncertain event will be formed and will lead to derivative events. 

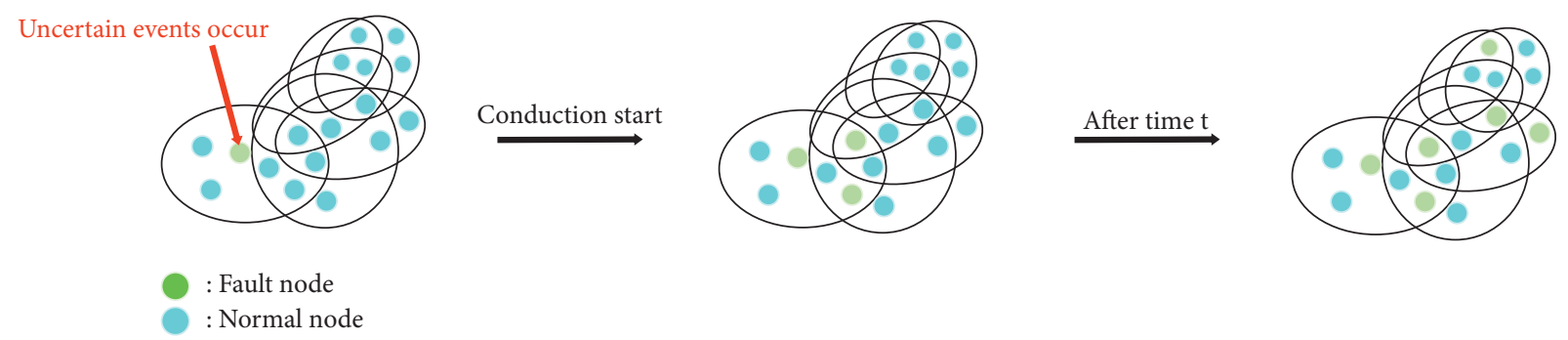

FIGURE 3: Example of network propagation of uncertain event.

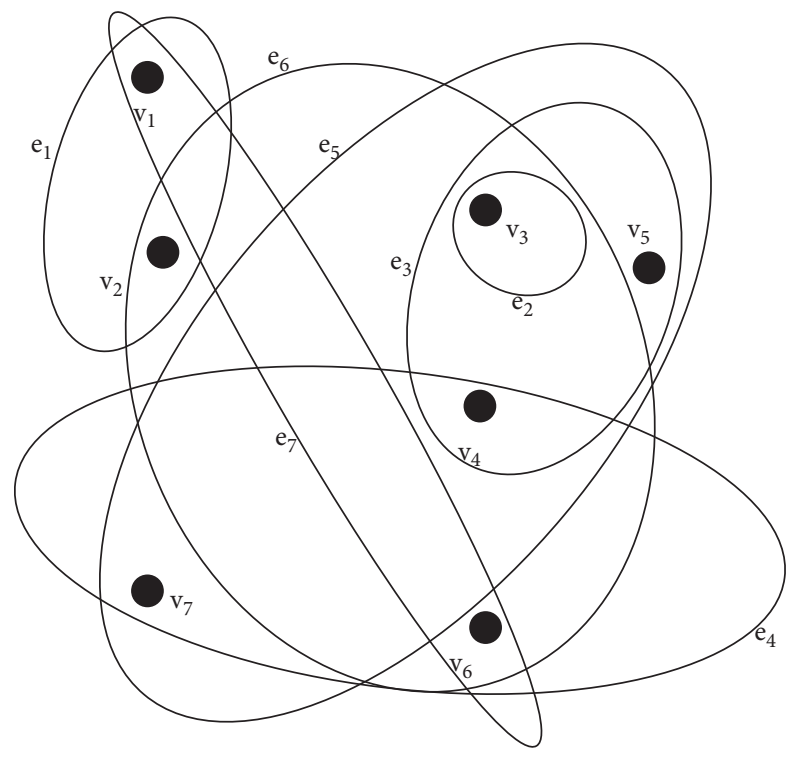

FIGURE 4: Graphical representation of hypergraph.

3.2.4. Hyperedge Hyperdegree. Hyperedge hyperdegree refers to the number of nodes contained in a hyperedge. In the uncertain event propagation network, it indicates the nodes' number that is affected by an uncertain event. The more nodes affected, the larger the influence range of this uncertain event. This index has important reference value for the stability and scheduling of the initial plan.

3.3. Modelling. The uncertain event propagation network model is used to describe the influence and diffusion of uncertain events in multilevel handlings at container terminal. Taking facilities as nodes and the uncertain events and their derivative events as hyperedges, a node can be affected by multiple uncertain events, and one uncertain event can also affect multiple nodes, and even some nodes will generate new derivative events after being affected. Therefore, by abutting these nodes, the propagation network model is constructed, and its schematic diagram is shown in Figure 5; the solid dots represent the old nodes, the hollow dots represent the newly added nodes, and the dashed curves represent the newly connected hyperedge.

The hypernetwork modelling algorithm is as follows.
3.3.1. Initialization. It is assumed that the hypernetwork initially has $m$ nodes $(V 1, V 2, V 3, \ldots, V m)$ and a hyperedge $E 1=\left\{V_{1}, V_{2}, V_{3}, \ldots, V_{m}\right\}$ containing $m$ nodes.

3.3.2. Hyperedge Growth. According to the process of container terminal handlings, the nodes involved in each uncertain event and its derivative events are neither a certain fixed value nor a certain equal probability, so the number of facilities (i.e., the number of nodes) is set to conform to Poisson distribution. Therefore, at time $t$, each time a new node $V$ is added, a random positive integer $L$ is produced by Poisson distribution probability:

$$
p(k, \lambda)=\frac{\lambda^{k}}{k !} e^{-\lambda}(k=0,1,2, \ldots),
$$

the new node $V$ and the $L(L \leq m)$ existing nodes in the network form a new hyperedge $E i=\{v i 1, v i 2, \ldots, v i L, v\}$.

3.3.3. Priority Connection. $L$ nodes are preferentially selected from the $M$ existing nodes in the hypernetwork according to the probability and form a hyperedge with the newly added nodes. The probability $W(i)$ of selecting the connected node $i$ is as follows:

$$
W(i)=\frac{d H(i)}{\sum_{i=1}^{n} d H(i)} .
$$

The molecular is equal to the number of hyperedges including node $i$, i.e., the degree of node $i$. The denominator is the sum of the degrees of all current nodes.

According to the above model construction process, the propagation hypernetwork model is analysed based on the mean field theory. In the initial hypernetwork, if there are $m$ nodes and a hyperedge, the initial degree of each node is 1 . Since one node is added in the network at a time, there are $m+t$ nodes and $t+1$ hyperedges in the hypernetwork after $t$ times. When a new node enters the network, the old nodes are selected to form a new hyperedge, which causes the nodes' hyperdegree change. The hyperdegree of the node $i$ should satisfy the dynamic equation:

$$
\frac{\partial d H}{\partial t}=p(k ; \lambda) W(i) .
$$




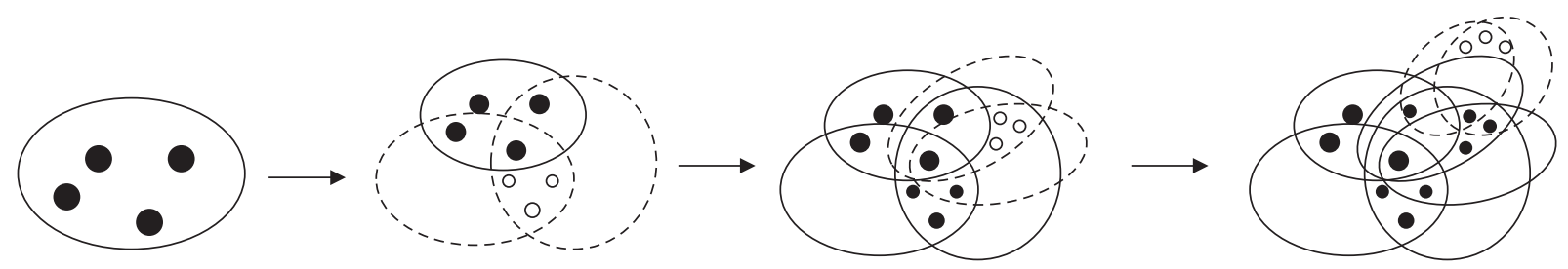

Figure 5: The evolution process of a hypernetwork.

After $t$ times, the sum of all nodes degrees is approximately

$$
\sum_{i=1}^{n} d H(i) \approx m+t(\lambda+1) .
$$

According to Poisson distribution theorem, the expected value and variance of Poisson distribution are both $\lambda$. That is, when $t$ is large enough, $p(k ; \lambda)$ approaches to $\lambda$. Therefore, by combining formula (2) and formula (4), formula (3) can be obtained:

$$
\frac{\partial d H}{\partial t}=p(k ; \lambda) W(i)=\lambda \frac{d H(i)}{m+t(\lambda+1)} \approx \frac{\lambda}{\lambda+1} \frac{d H(i)}{t} .
$$

When each node enters the network, the initial hyperdegree is 1 , and the solution of this partial differential equation is as follows:

$$
d H=\left(\frac{t}{t i}\right)^{\lambda / \lambda+1}
$$

Because the nodes with hyperedges in the hypernetwork are randomly selected, the probability that the nodes have hyperdegree is as follows:

$$
P(d H(t)<d H)=P\left(t i>\frac{t}{d H^{\lambda+1 / \lambda}}\right) .
$$

Assuming that at the same time interval, new hyperedge adding obeys uniform distribution, i.e., $t i$ has a constant probability density $p(t i)=1 / t$, and thus substituting it into formula (7) [12], we obtain

$$
P\left(t i>\frac{t}{d H^{\lambda+1 / \lambda}}\right)=1-P\left(t i \leq \frac{t}{d H^{1+(1 / \lambda)}}\right)=1-\frac{t}{d H^{\lambda+1 / \lambda} \cdot t^{\prime}} .
$$

Deriving formula (8), the hypernetwork hyperdegree distribution $P(d H, t)$ is as follows:

$$
P(d H, t)=\frac{\partial P(d H(t)<d H)}{\partial d H}=\frac{\lambda+1}{\lambda} d H^{-(2+(1 / \lambda))} .
$$

Through the derivation of formula (9), we can get the power law distribution of the node hyperdegree distribution function of the random hypernetwork constructed in this paper. And in the fourth part, the change of distribution function with different parameters $n$ and $m$ will be given by simulation [12].

\section{Simulation Analysis}

4.1. Risk Analysis of Uncertain Event Propagation Network Based on Hypernetwork. The risk analysis of uncertain events can better understand the impact of uncertain events at container terminal and help port authorities to strengthen the management of the important handling links and prevent uncertain events engendering greater impact. Therefore, the derivation and development of uncertain events can be better controlled and improved the operation efficiency [21].

In order to better explain the impact of uncertain events at container terminals, this section evaluates several types of uncertain events that occur frequently at container terminals through two indicators, the affected extent of nodes and the influence extent of uncertain events.

4.1.1. The Affected Extent of Nodes. The affected extent of nodes refers to the measurement of the derivative events that may occur after a node is affected by uncertain events. In the multilevel handling propagation network, there are many factors that may affect the initial plan, among which the main ones are as follows. (a) The number of uncertain events $(N)$, a node may be affected by multiple events, which can be reflected by the node hyperdegree. (b) Relevance between each handling $(R)$, which is the fundamental reason for the uncertain events to propagate in multilevel handlings. The more complex the correlation among handlings, the greater the probability of potential accidents. This index is reflected by the node degrees. (c) Derived event probability coefficient $(P)$. Due to the different extent of association among handlings, some nodes will not continue to trigger other events, while some nodes may become new uncertain events and trigger derived events. The probability coefficient of derived events has certain randomness and can be given according to statistical data. According to the above analysis, the affected extent of nodes can be defined as $S(V i)=N i * R i * P i$.

4.1.2. The Influence Extent of Uncertain Events. The influence extent of uncertain events refers to the comprehensive influence extent of all nodes affected by uncertain events, and the derivative of events should be considered at the same time, so its influence extent of uncertain events is defined as $S\left(e_{j}\right)=\alpha_{j} \sum_{i=1}^{M} S\left(e_{i}\right)$. 
Among them, $\alpha_{j}$ represents the coefficient of derivative events generated by uncertain events, and this index can be reflected by hyperedge. $M$ is the number of nodes that acted by an uncertain event, and it can be reflected by the degrees of edge $e j$.

By sorting out the literature considering the impact of uncertain events on container terminal handlings, the uncertain events and their derivative events that may occur at container terminal handlings are shown in Table 1:

The corresponding incidence matrix $L(G)$ is as follows:

$$
L(G)=\left[\begin{array}{lllllll}
1 & 0 & 0 & 0 & 0 & 0 & 1 \\
1 & 0 & 0 & 0 & 0 & 1 & 0 \\
0 & 1 & 1 & 0 & 1 & 1 & 0 \\
0 & 0 & 1 & 1 & 1 & 1 & 0 \\
0 & 0 & 1 & 0 & 1 & 0 & 0 \\
0 & 0 & 0 & 1 & 0 & 1 & 1 \\
0 & 0 & 0 & 1 & 1 & 0 & 0
\end{array}\right] .
$$

Table 2 shows a final calculation result about the affected extent of nodes. It can be seen that the two nodes with the higher value are $V 4$ (YC) and V3 (AGV). It represents that they have a higher correlation with other nodes. Therefore, it is necessary to focus on monitoring of these two nodes to reduce the occurrence of secondary and derivative events when scheduling.

Table 3 shows a final calculation result about the influence extent of uncertain events. It can be seen that the three hyperedges with the higher value are $E 6$ (equipment failure), E5 (truck arrival delay), and E3 (loading and unloading operation volume). It shows that they act on more nodes and further illustrate that they have a large influence range in the whole network. Therefore, the port authorities should pay attention to improving the flexibility of the operation plan of relevant nodes so as to more effectively and timely deal with the situation that the actual handling deviates from the plan when these uncertain events occurred.

The chain propagation of multilevel handlings in Figure 6 is based on the following rule: the nodes are deleted from high to low according to the affected degree of nodes shown in Table 2. From Table 2, the highest values of node $V 3$ and node $V 4$ indicate that these two nodes are vulnerable to uncertain events; it is necessary to avoid the connection among these nodes and other nodes as much as possible and at the same time ensure the connectivity of the graph. However, the chain propagation graph cannot further express more complex relationships with the increase in the number of nodes. Furthermore, the chain propagation will become cumbersome. Therefore, when considering the impact of uncertainties on specific nodes in multilevel handlings at container terminals, network propagation will be more applicable.

4.2. Simulation Analysis of Uncertain Event Propagation Network Based on Hypernetwork. According to the export process of containers terminal, the freight company arranges the truck to delivery. After entering the port, the truck arrives at the designated container area and unloads. Then, the container is stored into the yard by the YC. After that, AGV will send the container to the designated QC for loading operation. In this process, if the truck was delayed on the road, the loading operation of the ship will be delayed, and the scheduled sailing time of the ship will be delayed. On the other hand, it will further delay the ship arrival at the next transit port and affect the unloading operation and other operation plan at the next port. Even if the delayed container is arranged to be transported by other ships, the delay of the container will affect the loading and unloading operation in the next port.

In this whole process, it is only the delay of the truck arrival at first, but it constantly triggered the operation of other links change, and the influence continues to expand. It even further leads to the delay of the ship arrival at the next port. Taking Yangshan Port as an example, Yangshan Phase IV Automated Terminal currently has 16 QCs, 80 YCs, and 130 AGVs, the average annual container throughput is over 4 million TEUs, and equipment investment is expected to continue increasing in the future. The propagation and evolution of uncertain events among multilevel handlings are very serious at automated container terminals. Figure 7 shows the simulation results of distribution function under different parameters $n$ and $m$. The abscissa $d H(i)$ represents the node hyperdegree; hyperdegree refers to the number of hyperedges including node $i$, which can be used to measure the importance of node in hypernetwork. In the uncertain event propagation network, node hyperdegree indicates the number of uncertain events affecting nodes. The larger the hyperdegree is, the more vulnerable the node is. The vertical coordinate $P(d H)$ represents the hyperdegree distribution, and hyperdegree distribution $P(d H)$ reflects the level distribution that nodes be affected in the network.

According to simulation results, the probability distribution of node hyperdegree becomes smaller with the increase in node hyperdegree, and this change becomes more and more obvious with the increase in $n$. According to the definition of node hyperdegree, this shows that in the process of hypernetwork growth, the elder nodes are selected, the slower the growth rate of its hyperdegree distribution, i.e., the slower the growth rate of uncertain events affecting these nodes. This is because for the multilevel handling hypernetwork at container terminals, if more old nodes are selected, the overlapping probability of operation will be higher and the diffusion range of the influence caused by uncertain events will be limited. When the hypernetwork is dominated by new nodes, the interaction frequency among different operations is higher and the propagation range of uncertain events in the multilevel handlings at container terminals will be larger.

Through the above deduction, it can be concluded that the node hyperdegree distribution of the random hypernetwork constructed in this paper conforms to the power law distribution. Figure 8 shows the comparison between this hyperdegree distribution function and the power law distribution function deduced above when $\lambda$ takes different values (according to the above simulation results, the date, 
TABLE 1: List of uncertainty events and job nodes.

\begin{tabular}{lc}
\hline Uncertain events and their derivative events $(E i)$ & Affected job nodes $(V i)$ \\
\hline Ship arrival delay $E 1$ & Berth $V 1$, QC $V 2$ \\
The quantity of allocation QCs $E 2$ & AGV $V 3$ \\
Handling capacity $E 3$ & AGV V3, YC $V 4$, yard allocation $V 5$ \\
Changes in freight demand $E 4$ & YC $V 4$, truck $V 6$, gate congestion $V 7$ \\
Truck arrival delay $E 5$ & AGV $V 3$, YC $V 4$, yard allocation $V 5$, gate congestion $V 7$ \\
Equipment failure $E 6$ & QC $V 2$, AGV $V 3$, YC $V 4$, truck $V 6$ \\
Weather and other factors $E 7$ & Berth $V 1$, truck $V 6$
\end{tabular}

TABLe 2: Analysis of the affected extent of nodes.

\begin{tabular}{lcccc}
\hline Node & Node hyperdegree $N$ & Node degree $R$ & Subhead-derived event probability coefficient $P$ & $S(V i)$ \\
\hline$V 1$ & 2 & 2 & 0.2 & 0.8 \\
$V 2$ & 2 & 4 & 0.7 & 5.6 \\
$V 3$ & 4 & 4 & 0.7 & 11.2 \\
$V 4$ & 4 & 5 & 0.8 & 16.0 \\
$V 5$ & 2 & 3 & 0.4 & 2.4 \\
$V 6$ & 3 & 5 & 0.4 & 6.0 \\
$V 7$ & 2 & 4 & 0.5 & 4.0 \\
\hline
\end{tabular}

TABLE 3: Analysis of influence degree of uncertain events.

\begin{tabular}{lccc}
\hline Hyperedge & $\begin{array}{c}\text { Hyperedge degree } \\
\alpha j\end{array}$ & $\begin{array}{c}\text { Hyperedge hyperdegree } \\
\text { Sy }\end{array}$ & \\
\hline E1 & 2 & 2 & 12.8 \\
E2 & 3 & 1 & 33.6 \\
E3 & 4 & 3 & 118.4 \\
E4 & 4 & 3 & 104.0 \\
E5 & 4 & 4 & 134.4 \\
E6 & 6 & 4 & 232.8 \\
E7 & 3 & 2 & 20.4 \\
\hline
\end{tabular}

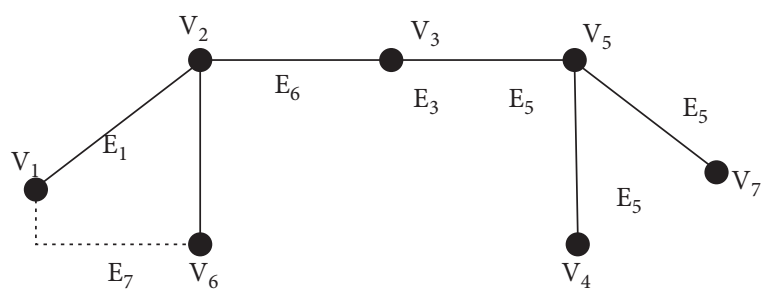

FIgURE 6: Chain propagation of multilevel handlings.

$n=10000$, and $m=5$ were chosen). According to Figure 8, when $\lambda=4$, the two figures can basically achieve coincidence.

\subsection{Comparative Analysis of Different Container Terminals.} In order to further verify the rationality of the proposed model, we compare two different container terminals, Xiamen Yuanhai Terminal and Shanghai Yangshan Phase IV Terminal. Xiamen Yuanhai Terminal is equipped with 3 automatic double-trolley QCs, 16 YCs, 18 AGVs, and 8 automatic container transfer platforms, with a total of 45 multilevel handling facilities. According to the project planning, Shanghai Yangshan Phase IV Terminal is expected to be equipped with 130 AGVs, 26 QCs, and 120 YCs, with a total of 276 multilevel handling facilities. In the setting of initial network nodes, this work assumes that there are three nodes in the initial network for Xiamen Yuanhai Terminal, while Shanghai Yangshan Phase IV Terminal is set to have five nodes, and the probability distribution diagram of node hyperdegree is shown in Figure 9. The propagation of uncertain events in the multilevel handlings at container terminals depends on the connections among the interactively coupled handlings. The propagation network scale expands with the increase in the number of hyperedges and nodes.

Figure 9 shows an obvious power law attenuation trend, which indicates the uncertain event propagation networks in the multilevel handlings at container terminals have scalefree features. It also reveals two other basic characteristics of the uncertain event propagation networks: "growth" and "preferred connection," which mean that nodes with larger hyperdegree will be preferred when the influence of uncertain events is propagated. However, due to the limitation of network scale, the speed of propagation will gradually tend to be flat; that is, with the increasing hyperdegree, the distribution of hyperdegree will become smaller and smaller, as displayed in two different terminals. This characteristic of Shanghai Yangshan Phase IV network with more nodes is more obvious, as shown in Figure 9 (2), while the Xiamen Yuanhai network with less nodes will show this characteristic faster, as shown in Figure 9 (1). This is because the number of selected nodes is random when selecting nodes to form a hyperedge in each time step, which makes the number of nodes included in the hyperedge change accordingly. However, for smaller networks, due to the limitation of node's number, the number of old nodes in the hyperedge will be more and more in the later period of network growth, so it will show scale-free characteristic faster. Based on the abovementioned characteristics of hypernetwork, it also reveals that port authorities need to strengthen the maintenance of some facilities with high node 

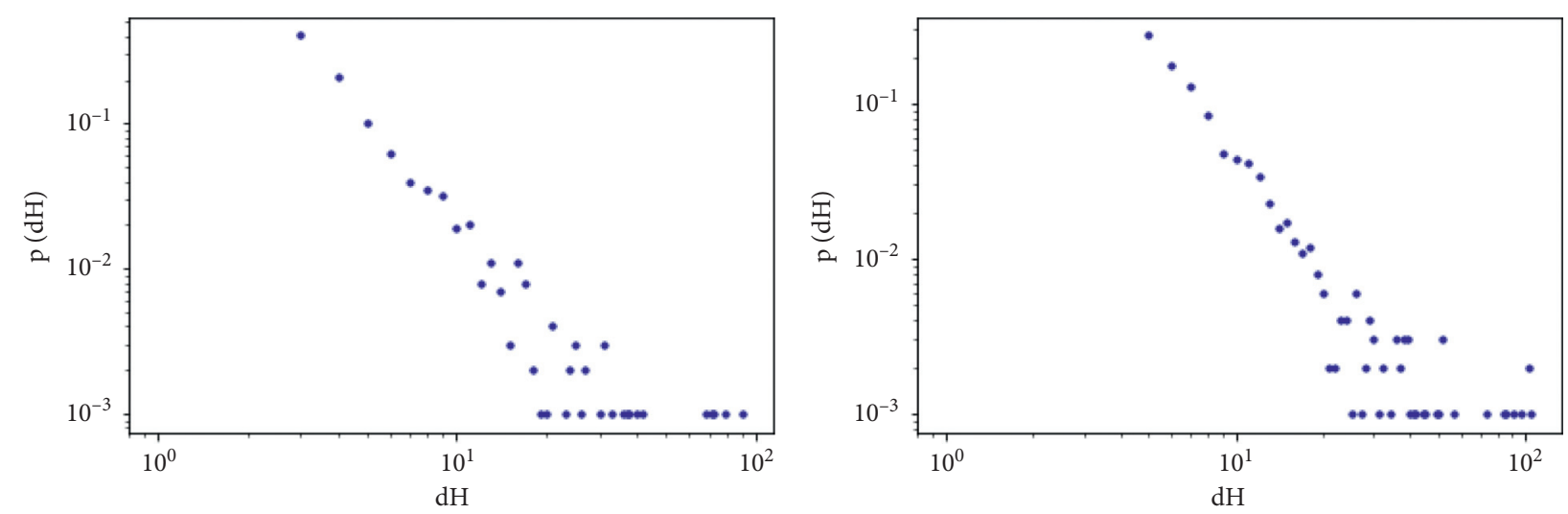

- $n=1000, m=3$

$$
\text { - } n=1000, m=5
$$

(a)

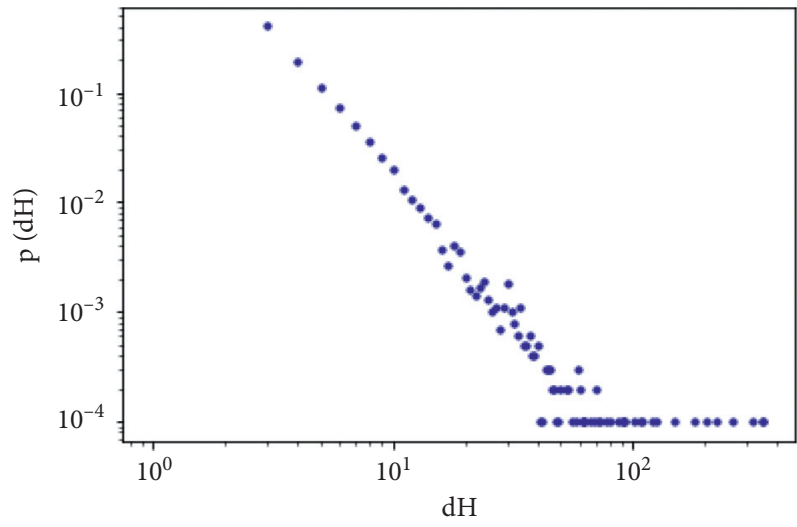

- $n=10000, m=3$

(c)

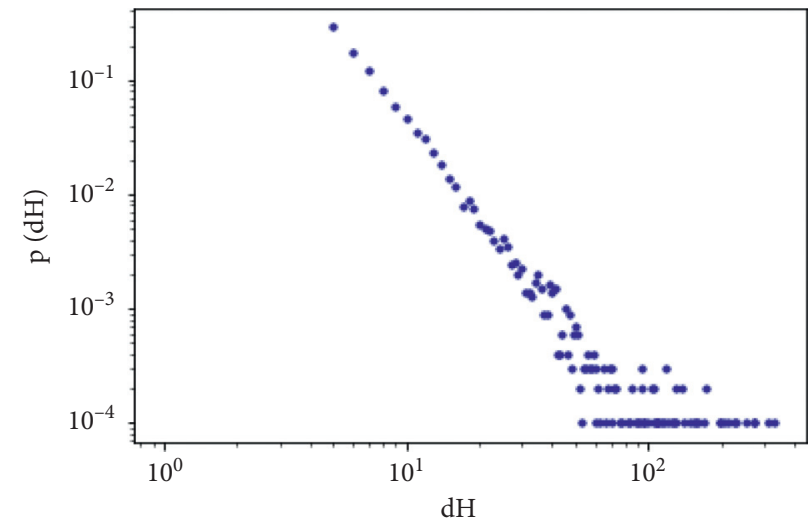

- $n=10000, m=5$

(d)

Figure 7: Double logarithmic hyperdegree distribution diagram.
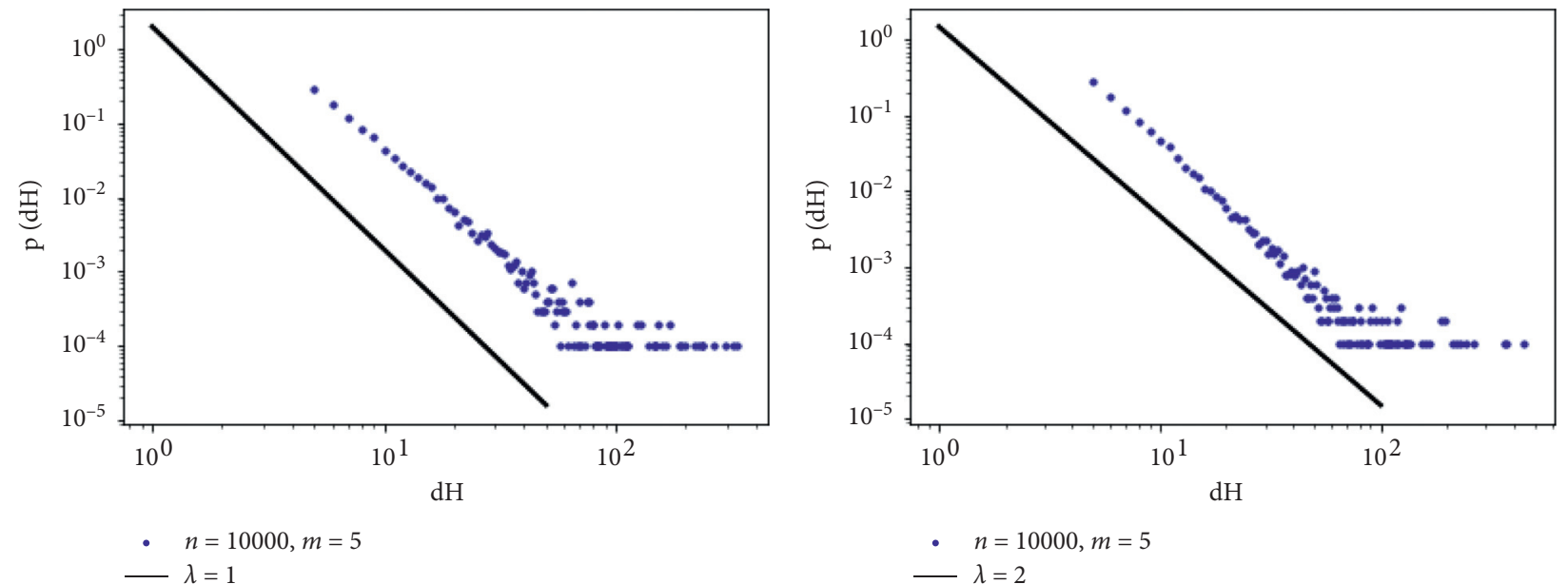

(a)

(b)

Figure 8: Continued. 


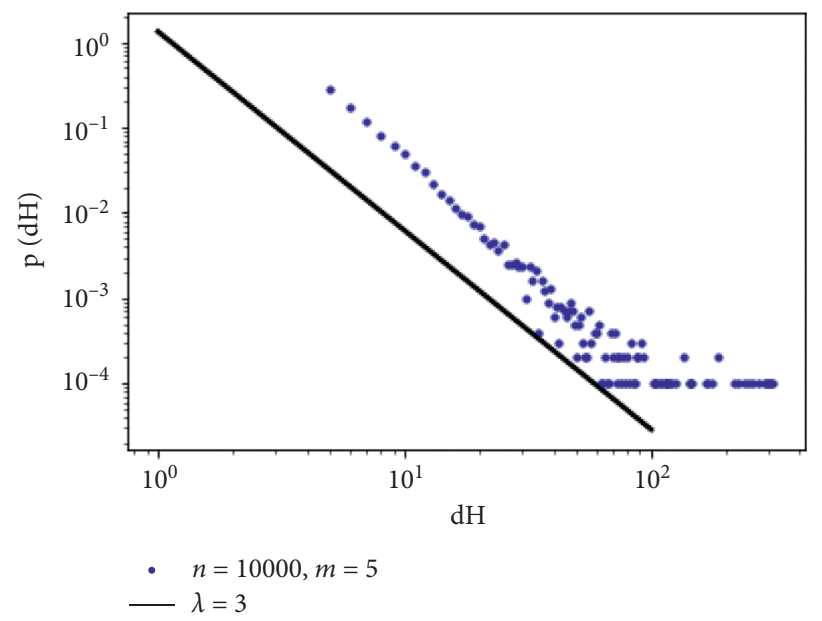

(c)

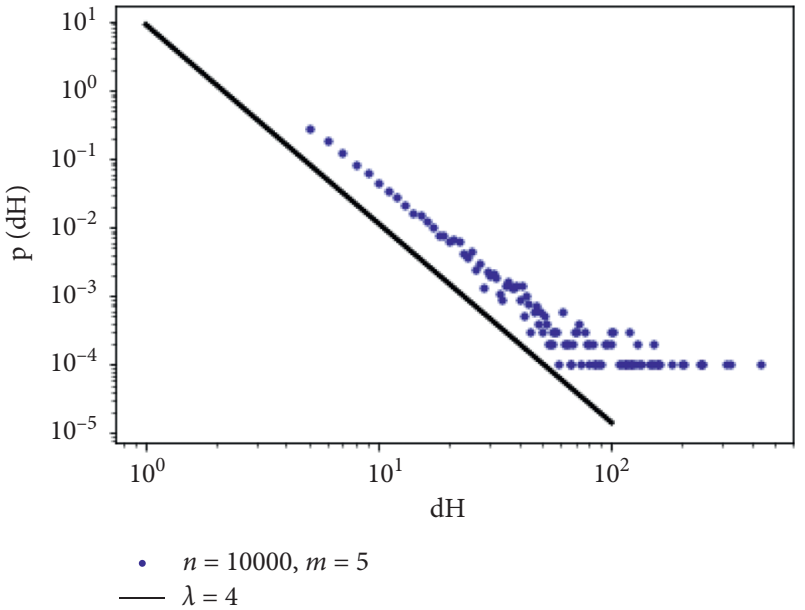

(d)

Figure 8: Simulation and theoretical analysis of double logarithmic hyperdegree distribution diagram.

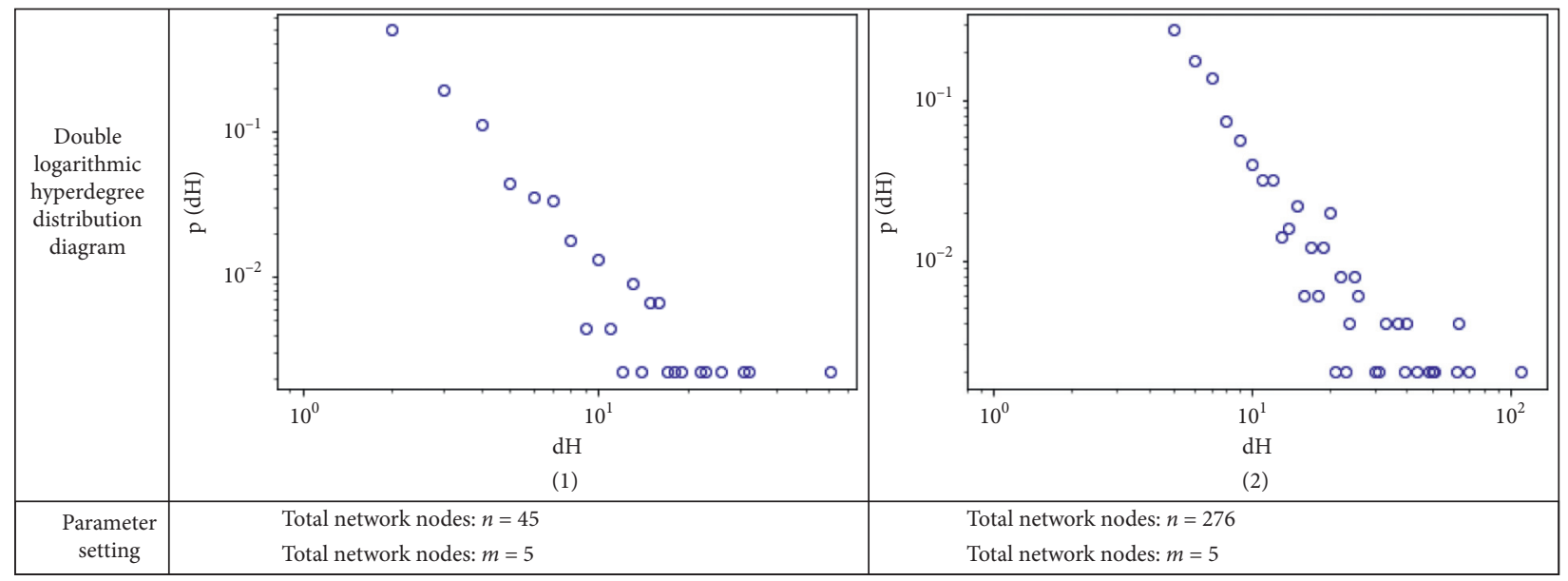

FIgURE 9: Hyperdegree distribution diagram of different terminals.

hyperdegree in daily operation so as to prevent such nodes from affecting more operations when uncertain events occur. At the same time, it is necessary to effectively manage equipment resources for some nodes with small hyperdegree, and this kind of resources will easily become the boundary of propagation range when uncertain events occur because nodes with high hyperdegree will use this kind of nodes to spread the influence continuously.

\section{Conclusion}

In this paper, the propagation influence of uncertain events in multilevel handlings at container terminals is analysed from the perspective of hypernetwork, and the container terminals are considered from the perspective of hypernetwork. At present, most of the related research studies considered uncertain events focused on a specific handling link, which could not show and analyse the correlation among operation nodes and the propagation impact of uncertain events in the whole container terminal. In view of this, this paper uses the hypernetwork theory to construct the uncertain event propagation network model in multilevel handlings at container terminals. Through the topological characteristics of the propagation network, the risk analysis is carried out, and the influence extent of some uncertain events and related nodes at container terminals are evaluated. Finally, the propagation changes of uncertain events at container terminals are analysed by simulation. The model has the following advantages:

(1) The topological characteristics of uncertain event propagation network are analysed by using the hypernetwork theory, which provides a powerful evaluation reference for analysing the correlation in the multilevel handlings and the probability of the secondary events. It is of certain value to reduce the impact of uncertain events on the initial plan arrangement, the occurrence of derivative events, and the possible losses caused by changes in the terminal plan so as to improve the handling efficiency of terminals. 
(2) For the model given in this paper, by reducing or increasing the nodes and hyperedges in the network, it can form different scales' network to do a further study on the optimization of handling efficiency at container terminals.

However, the uncertain events considered in this paper are mainly a rough summary of the existing literature and other materials, and more specific situations will occur in reality, so further refining the problems and obtaining more accurate and effective analysis will be the research focus in the next stage. This paper is only a preliminary attempt to apply hypernetwork to study the propagation network of uncertain events at container terminals, and more problems such as network topology have not been discussed in depth in this paper, which will be an important direction to do a further exploring in the future.

\section{Data Availability}

The data used to support the findings of this study are included within the article.

\section{Conflicts of Interest}

The authors declare that they have no conflicts of interest regarding the publication of this article.

\section{Acknowledgments}

This work was supported by the National Social Science Foundation Project of China (no. 17CGL018).

\section{References}

[1] M. R. Othman, J. Jeevan, and S. Rizal, "The Malaysian intermodal terminal system: the implication on the Malaysian maritime cluster," International Journal of E-Navigation and Maritime Economy, vol. 4, pp. 46-61, 2016.

[2] G. Tasoglu and G. Yildiz, "Simulated annealing based simulation optimization method for solving integrated berth allocation and quay crane scheduling problems," Simulation Modelling Practice and Theory, vol. 97, Article ID 101948, 2019.

[3] L. Xiang, C. Liu, and L. Miao, "Reactive strategy for discrete berth allocation and quay crane assignment problems under uncertainty," Computers \& Industrial Engineering, vol. 126, no. DEC, pp. 196-216, 2018.

[4] O. Postolache, Y. Yang, and Y. Zhou, "Adaptive autotuning mathematical approaches for integrated optimization of automated container terminal," Mathematical Problems in Engineering, vol. 2019, no. 4, 14 pages, Article ID 7641670, 2019.

[5] M. Ma, H. Fan, M. Ji, and Z. Guo, "Collaborative optimization of truck delivery reservation and yc scheduling in container terminal," Journal of Transportation, vol. 3, pp. 202-209, 2018, in Chinese.

[6] M. Mohammadi and K. Forghani, "Solving a stochastic berth allocation problem using a hybrid sequence pair-based simulated annealing algorithm," Engineering Optimization, vol. 51, no. 10, pp. 1-19, 2019.

[7] T. Yu, Z. Qiang, and Z. Benfei, "A genetic algorithm based on spatiotemporal conflict between continuous berth-allocation and time-varying specific crane assignment," Engineering Optimization, vol. 51, no. 3, pp. 390-411, 2019.

[8] Z. Wang and C. Guo, "Minimizing the risk of seaport operations efficiency reduction affected by vessel arrival delay," Industrial Management \& Data Systems, vol. 118, no. 7, pp. 1498-1509, 2018.

[9] H. P. Hsu, T. L. Chiang, C. N. Wang et al., "A hybrid GA with variable quay crane assignment for solving berth allocation problem and quay crane assignment problem simultaneously," Sustainability, vol. 11, no. 7, 2018.

[10] D. N. Prayogo, A. Hidayatno, and Komarudin, "Developing a robust optimization model for seaside operations in container terminal under uncertainty environment," IOP Conference Series Earth and Environmental Science, vol. 235, 2019.

[11] Y. Xie and D. P. Song, "Optimal planning for container prestaging, discharging, and loading processes at seaport rail terminals with uncertainty," Transportation Research Part E: Logistics and Transportation Review, vol. 119, 2018.

[12] F. Hu, Research on the Structure, Modeling and Application of Complex Hypernetwork, Shaanxi Normal University, Xi'an, China, 2014, in Chinese.

[13] Y. Chi, X. Tang, Y. Lian et al., "A supernetwork-based online post informative quality evaluation model," Knowledge-Based Systems, vol. 168, pp. 10-24, 2019.

[14] S. Kan, W. Lv, and F. Guo, "Dynamic learning super network modeling of a complex product system based on multi-organization cooperation," Modern Physics Letters B, vol. 32, no. 31, 2018.

[15] H. Pu, Y. Li, C. Ma et al., "Analysis of the projective synchronization of the urban public transportation super network," Advances in Mechanical Engineering, vol. 9, no. 6, 2017.

[16] W. Xiao and L. Wang, "The identification of maritime industry clusters using maritime industry super-network," Ekoloji Dergisi, vol. 107, 2019.

[17] Z. Liu, W. Chen, C. Zhang, C. Yang, and H. Chu, "Data supernetwork fault prediction model and maintenance strategy for mechanical product based on digital twin," IEEE Access, vol. 7, pp. 177284-177296, 2019.

[18] C. F. Zhu, Z. K. Zhang, and C. X. Ma, "Research on emergency logistics dynamic network based on super network," Latin American Applied Research, vol. 47, no. 1, pp. 11-16, 2017.

[19] Y. Su, J. Qin, P. Yang et al., "A supply chain-logistics supernetwork equilibrium model for urban logistics facility network optimization," Mathematical Problems in Engineering, vol. 2019, Article ID 5375282, 12 pages, 2019.

[20] Y. Ji, C. Wu, S. Yang, M. Guo, and Y. Zhang, "Chain evolution model of network security events based on hyper-network," Journal of Cyber Security, vol. 4, no. 1, pp. 89-100, 2019, in Chinese.

[21] X. Wang and L. \&Gui, "Research on the evolutionary network of sudden disasters based on hypergraph," Journal of Mathematics in Practice and Theory, vol. 12, p. 7, 2019, in Chinese. 